\title{
Stabilization of vibrations of rotary machines in radial sliding bearings with integrated liquid
}

\author{
Aleksandr Kiryukhin ${ }^{1, *}$ \\ ${ }^{1}$ Nakhimov Naval School, Department of Ship Power Plants Exploitation, 1A, Dybenko str., Sevastopol, \\ Russia, 299053
}

\begin{abstract}
The use of radial bearings with segments on a self-generated hydrostatic suspension has been substantiated in order to satisfy the requirements for precision positioning of axes and efficiency of stabilization of shaft oscillations of power-consuming rotary machines. The methods of turbomachine dynamical analysis based on the modelling of the viscous friction forces process and inertia in lubricating films that balance the harmonic loads on the shaft by redistributing the lubricant flow parameters in the bearing gaps with integrated liquid dampers have been further developed. The complex approach for improving functional properties of turbomachine supporting block on the basis of multicriteria optimization of hydraulic tracts parameters and lubrication system operating modes is presented.
\end{abstract}

One of the main trends in the development of stationary and transport energy is the increase in the productivity of rotary machines, while increasing the resources and reducing the costs of production and operation. With regard to ship turbomachines, the consequence is an increase in the specific powers and rotational speeds of the main and auxiliary turbine units, accompanied by an increase in the masses and overall size of the rotors and, correspondingly, the increase in the forces and their moments acting in the rotational parts, the development of oscillations and the growth of mechanical losses when generation, transformation and transmission of energy on board. Durability and reliability of rotary machines, in the first place, depend on the operation mode of tribocoupling in supporting blocks. Under the operational factors influence, tribomechanical and rheological processes are activated in the bearings, which contribute to dissipation of the supplied mechanical energy as to destructive mechanisms that cause the nutation of the rotor and the wear of the support nodes, and the protective ones in the implementation of which the resource for improving the operability of the machines is laid.

Among the directions for improving which are traditionally used sliding bearings in power engineering, constructive measures to prevent the cases of rotors self-oscillations are dominated by modifying the geometry of the friction surfaces (the use of lubricating grooves of various configurations and sizes, the creation of several lubricating wedges, etc.) and due to the mobility of the sliding surfaces $[1-11]$. The low load-bearing capacity at start-up conditions, the appearance and development of subharmonic oscillations on lubricating wedges limit the use of two-, three- and multi-cylinder bush bearings in propulsion engines $[5,6,7]$,

\footnotetext{
*Corresponding author: alkiryukhin@yandex.ua
} 
and the critical dependence of hydrostatodynamic supporting blocks against the lubricant supply pressure prevents their expansion on the board of sea transport.

In bearings with movable inserts on point supports, self-installation of segments in the circumferential and axial direction facilitates the alignment of the pressure diagrams on their principle surfaces and the intensification of the recreational reactions of the lubricating layer to destabilizing the shafts movable balance under the influence of structural, mounting and operational factors. However, with an increase in the number of inserts, the bearings vibration resistance can deteriorate due to the development of self-excited oscillations of the unloaded segments [1]. To partially eliminate this effect, especially at higher rotor speed, the multi-segment bearings with a reduced installation gap are used, and to ensure thermodynamic and structural stability of the lubricating layer low-viscosity lubricants are used (water condensate with special inhibitor additives, aqueous solutions of polymers , cryogenic liquids and inert gases).Moreover, the gas lubricant is successfully used in radial bearings with a specific load up to $1.2 \ldots 1.4 \mathrm{kN} / \mathrm{cm}^{2}$. At the same time, the desoisition of such supporting blocks to the rising of instability of the type of "half-speed vortex" [5] requires comprehensive reasonableness and careful calculation argumentation of the solutions for the modernization of turbomachines with their application.

The use of elastic and flexible slip launcher avoids the reduction of productivity with the development of the resource, supports the necessary bearing tension and provides operation in a narrower range of variation of the stiffness and damping coefficients. Their wide use for stabilizing the vibrations in turbomachines is still maintained due to relatively high cost of the precision of design and production, the difficulty in determining the actual gaps on variable loads and poor damping on the critical the velocity $[8,12]$.

However, the promising cantilever-turning construction [13] allows to hold the shaft effectively in the supporting block at low speeds, minimizing the radial ascent of the stud in the space of the bearing gap, which influences the centering and vibration stability of the rotor in some high-performance turbomachines. The addition of such supporting blocks with hydrodynamic film dampers (Fig. 1, a) allows to adjust the stiffness and damping practically independently of each other, which makes ISFD ${ }^{\circledR}$ technology of the WaukeshaBearings company [14] one of the leaders in production solutions for controlling and monitoring the vibration of rotary machines. While conventional Squeezing Film Dampers (SFD) realize the effect of the dynamic rigidity of the hydrobibois isolators, which depends on the amplitude and frequency of the oscillations, in the ISFD ${ }^{\circledR}$ structure, the stiffness is determined only by S-shaped springs. This allows to ensure accurate predictability and effective rebuilding of critical speeds and turbomachines operating modes, to reduce the gain factor independently of the amplitudes and frequencies of the forced and self-excited oscillations of the rotor system, to increase the service period of the bearings by the run-out and fatigue of the babbitt layer.

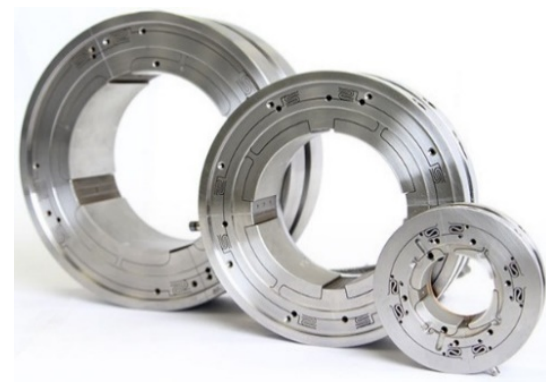

a
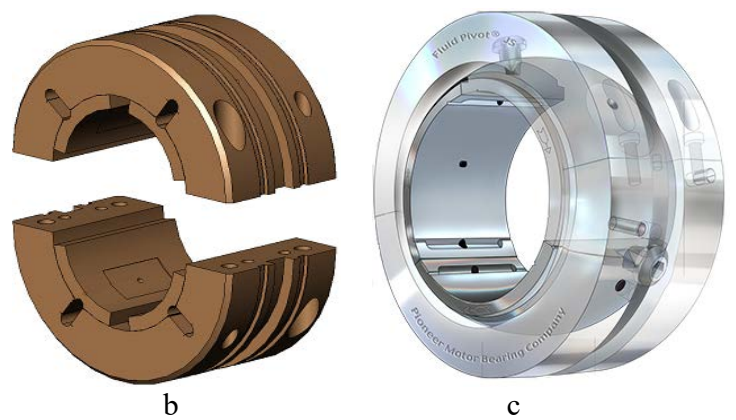

Fig. 1. Constructions of hydrodynamic bearings with ISFD ${ }^{\circledR}$ by dampers and inserts on flexible supports (a), with inserts on hydrostatic suspension from an external source (b) and on a self-generated hydrostatic suspension (c) 
The concept of integration of the bearing and the damper in one device is realized in the radial bearings constructions with inserts with the hydrostatic suspension from an external source (Fig. 1, b) and with segments on a self-generated hydrostatic suspension (Fig. 1, c). Their operative concept is based on hydrostatic support of self-installing inserts in the gap. Under the influence of hydrostatic pressure, the segments float up, follow the oscillations of the shaft and effectively damp them in a wide amplitude-frequency range by the viscosity forces and inertia of the hydrostatic layer. According to [15], when installing the rotor in supporting blocks with inserts on the hydrostatic film, the resonance peak at the first critical frequency is reduced in $2 \ldots 3$ times in comparison with the rotor on five-segment bearings. Accordingly, the damping coefficients of supporting blocks with inserts on the hydrostatic suspension are 2.1 ... 2.2 times higher than for five-segment bearings, while the stiffness coefficients of both types of bearings are the same and vary in the range $(0.2 \ldots 2.5) \cdot 10^{8} \mathrm{~N} / \mathrm{m}$ depending on the rotor speed.

Adaptive self-regulation of the dissipation processes of rotor vibration energy is especially characteristic for bearings with a hydrostatic layer formed by removing a part of the lubricant from the gradient velocity flow on the working surface of the inserts into the pockets located on their rear sides. When the oscillations intensify, the shear movement increases the oil supply of the hydrostatic layer, and the vibration energy is effectively dissipated when the lubricant is throttled in the bypass channels into the hydrostatic pockets. The wide end sections on the contours of the hydrostatic pockets stimulate a rapid increase in the carrying capacity of the intensively replenish hydrostatic layer due to the inertia of the viscous lubricant flow in the slot-type gap under the most loaded inserts.

The bearing capacity and rigidity of the hydrodynamic bearing layer, which is increasing due to lifting and self-installation of the inserts, stabilizes effectively the trajectory of the mobile balance of the spike and reduces the rotor swing. To minimize the gyroscopic effects, the company "PioneerMotorBearingCo" proposed the design of bearings with the backs of spherical inserts [16], facilitating the self-installation of segments in the axial direction and preventing their flagellation.

Thus, the requirements for the accuracy of axle retention and the stabilization of the oscillations of the shafts of marine power-consuming rotor machines are satisfied the best by sliding supporting blocks with integrated liquid damper: segment and bushing ISFD ${ }^{\circledR}$ bearings, as well as hydrodynamic bearings with segments on a self-generating hydrostatic suspension. Essentially they are elastic-damper supporting blocks and they allow to reduce the vibration level of rotors several times regardless of their nature $[14,15]$.

The stability of the movement of machine rotors in sliding bearings is affected by a number of design, technological and operational factors, in particular, the ability of lubricating films separating friction surfaces to adapt to changing operating conditions. The parameters of the orbital motion of the shafts in the supporting blocks are determined by the transformation regularities of mechanical energy when liquid friction and depend on the nature and intensity of energy dissipation processes in thin lubricating films that compensate quasistationary and harmonic loads on the rotor system. This circumstance requires the development of models and methods for calculating the dynamics of ship rotary systems, developing scientifically valid recommendations for the design of turbomachinery and ensuring their operability, which is lost during the intensive marine vehicles operation.

To establish the parameters of the synchronous precession of a rotor in sliding bearings with ISFD ${ }^{\circledR}$ dampers, the authors of the work $[12,13,14]$ propose to use a generalized model of dynamic reactions of an isotropic lubricating layer described by the Navier-Stokes equations. The damper is modeled by a centering ring, the hydrodynamic forces in which are found by integrating the pressure distribution function from the modified Reynolds equations [11].The dependence of coefficients of stiffness and damping of bearings on the size of the working gap, viscosity of the lubricant, shaft rotation speed, support geometry and damper 
were obtained by analytical and analytical methods of L. SanAndrés. However, such an approach to the decomposition of the problems of modeling the reactions of elastic-damper supporting blocks does not give reliable results in the calculation bearings with inserts on the hydrostatic suspension, the mobile balance of spikes in the gaps of which is established by self-regulating redistribution of pressures, temperatures and expenses of working fluids in the cojoint hydrodynamic and hydrostatic layers.

Processes of interaction of viscous friction forces and inertia in lubricating films that balance harmonic loads on the shaft by redistributing the parameters of the flow of working fluid in the gaps of the studying supporting blocks are formalized in the form of a mathematical model based on the solution of the system of rotor dynamics equations [17], which is supplemented by the equations of conservation of momentum, mass and energy for the characteristic sections of the hydraulic bearings tract [18]. For the numerical solution of discrete analogs of the initial nonstationary equations, the method of integral identities is used: the computational domain is divided into a finite number of non-overlapping control volumes, to calculate the integrals between the nodal points of which the approximation of the desired function is used according to the scheme described inthe work [19].

In the iterative calculation of pressure distributions, temperatures, thicknesses of the hydrodynamic and hydrostatic pressure layers in the chambers on the inner and outer surfaces of the segments, they are considered equal unknown constants, which are refined at each iteration of the equation of the lubricant consumption balance. The position of the segments in the bearing gap is determined from the equations of their motion, based on the equilibrium of the hydrodynamic and hydrostatic layers reactions on the change in the position of the shaft in the supporting block. The substitution of the lubricant layer reactions at each time step into the equations of the rotor motion makes it possible to establish the trajectories of its vibration displacement.

The multi-variant calculation of the developed model has established that under conditions of unfavorable stresses in round cylindrical bearings, the use of supporting blocks with integrated liquid dampers activates adaptive processes in the lubricating layer that effectively reduce the intensity of the destabilizing effect of structural and operational factors, and improve the accuracy of retention of shaft axes (Fig. 2). In view of the gradients identical effect of thermohydrodynamic and kinematic parameters on the elastic-damping properties of the bearing lubrication layer, the task of complex optimization of work processes is brought to the search for extremes of the efficiency index of the use of the working capacity of the tribosystem elements:

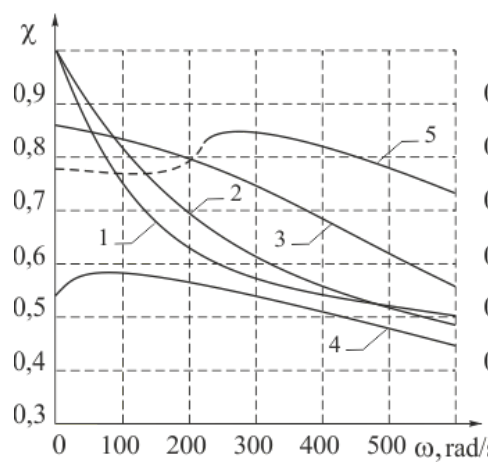

a

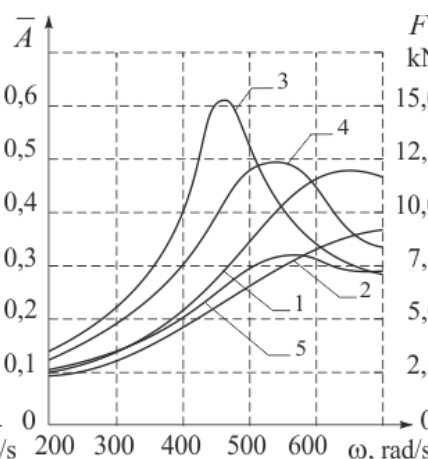

$\mathrm{b}$

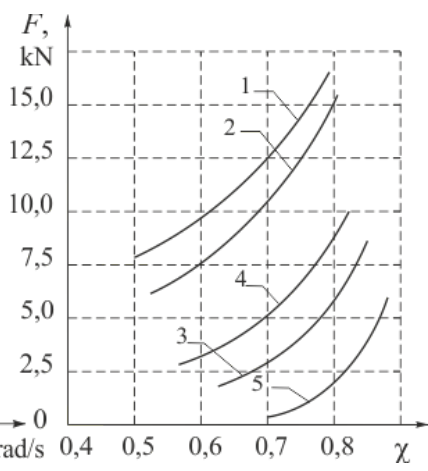

c

Fig. 2. Parameters of retention processes of the rotor of a steam turbine generator in radial slip bearings $\left(\mathrm{N}_{\mathrm{e}}=3 \mathrm{MW}, \mathrm{R}=0.05 \mathrm{~m}, \omega=314\right.$ radians $\left./ \mathrm{s}\right)$ : a - relative shaft eccentricity in the hydrodynamic bearing on turbine oil $\mathrm{T}_{\mathrm{r}}-46(1)$, and the bearing with the inserts on the hydrostatic suspension (2), in the hydrostatodynamic bearing (HSDB) bearing on Aquanole 7 (5: the dashed line - the operation of the 
rotor hydro-lifting system), the HSDB on water lubrication with 6 rectangular narrow chambers (3), HSDP on an aqueous solution of polyhexamethyleneguanidine phosphate with 4 double-T chambers(4); $\mathrm{b}$ - the amplitude of relative shaft oscillations $\left(A=A / h_{\min }\right) ; \mathrm{c}$ is restoring reactions to the dynamic bearing loading.

$$
D_{u}=\frac{d_{\chi}-d_{\Sigma}}{d_{\chi}}
$$

where $d_{\chi}$ is the dimensionless desirability parameter corresponding to the minimum deviation of the shaft axis from the calculated deviation by the given load-speed characteristics; $d_{\Sigma}=f\left(k_{F}, k_{Q}, k_{f}, k_{e}, k_{e s}, k_{y}\right)$ is a dimensionless parameter that takes into account the degree of perfection of the system in terms of load capacity $\left(\mathrm{k}_{\mathrm{F}}\right)$, lubricant consumption $\left(\mathrm{k}_{\mathrm{Q}}\right)$, friction losses $\left(\mathrm{k}_{f}\right)$ and energy costs for work $\left(\mathrm{k}_{e}\right)$, ecological safety $\left(\mathrm{k}_{e s}\right)$ and stability of rotor mobile balance $\left(\mathrm{k}_{\mathrm{y}}\right)$.

The coefficient of load-carrying capacity is optimized by the criterion of minimizing the deviations in the position of the shaft axes in the bearings, taking into account the energy efficiency and the vibration stability of the rotor system given by the dependencies of the type [2]:

$$
k_{Q}=\frac{F}{Q} \cdot \frac{\pi h_{0}^{3}}{8 \mu R L}, \quad k_{f}=\frac{F}{N_{f}} \cdot \frac{\mu \omega R^{2}}{p_{0} h_{0}}, k_{e}=\frac{F}{N_{f}+N_{Q}} \cdot \frac{\pi h_{0}^{3} p_{0}}{8 \mu R L}, k_{y}=\sqrt{\frac{4 p_{0} R L}{\omega_{y} m h_{0}}},
$$

where $h_{\mathrm{o}}, L$ is the radial gap and the length of the bearing inserts; $\mu$ - viscosity of the lubricating material; $m, R, \omega$ - mass, radius and angular speed of the shaft rotation; $F$ - load capacity; $Q$ - lubricant consumption; $N_{f}, N_{Q}$ - power on friction and pumping of lubricating material; $p_{o}-$ pressure in the pressure line of the lubrication system; $\omega_{y}$ - the stability limit of shaft rotation.

\section{Conclusion}

Tougher requirements for vibration resistance, energy efficiency and wear resistance of powerconsuming rotary machines are the most satisfied by sliding supporting blocks with synthesized liquid dampers. Within the multicriteria optimization task to minimize deviations of the position of the shaft axes in the bearing gaps with inserts on the hydrostatic suspension, significant factors have been identified and the ranges of the constructive parameters of the bearings and operating mode of the lubrication system have been determined, in which $25 \ldots 65 \%$ of the increase accuracy of axle shaft holding is achieved, 1.5 ... 2-fold decrease in vibration amplitudes and 10 ... $15 \%$ decrease in energy costs for retention of machine rotors in comparison with traditionally used segment bearings with self-installing inserts on rigid supporting blocks.

The priority direction of further research is improving the accuracy of retaining rigid and flexible rotors of power plants in hybrid and combined supporting blocks, bearings operating on fluid and gas lubrication, electromagnetic suspensions, improving the scientific basis for their trouble-free operation.

\section{References}

1. V.Ya. Kalmens, Providing vibro-reliability of rotary machines on basis of similarity and modeling methods (Publ. House SZPI, St.Pb, 1992) 
2. N.P. Artemenko, A.I. Chayka, V.N. Dotsenko, Hydrostatic supports of rotors of highspeed machines (Publ. House "Osnova", Kharkov, 1992)

3. V.A. Maksimov, G.S. Batkis, Tribology of liquid friction bearings and seals of highspeed turbomachines (Publ. House "FEN", Kazan, 1998)

4. L.A. Savin, O.V. Solomin, Modeling of rotor systems with liquid friction bearings (Mech. Engineering, Moscow, 2006)

5. K.V. Frolov, Modern tribology: Results and Prospects (Publ. House LKI, Moscow, 2014)

6. D. Childs Turbomachineryrotordynamics: phenomena, modeling and analysis (WileyInterscience, NY, 1993)

7. J. Frene, D. Nicolas, B. Berthe, M. Godet, Hydrodynamic Lubrication. Journal and Thrust Bearings (Tribology Series, 33, Elsever,1997)

8. W. J. Chen, E. J. Gunt, Introduction to dynamics of rotor-bearing system (Trafford publ., Bloomington, 2005)

9. A. Muszynska, Rotordynamics (CRC Press, Raton, 2005)

10. G. Genta, Dynamics of rotating systems (Springer, Berlin, 2005)

11. I.I. Kudish, M.J. Covitch, Modeling and analytical methods in tribology (CRC Press, Raton, 2010)

12. A.M. Rimpel, Analysis of flexure pivot tilting pad bearings with different damper configurations (A\&M University, Texas, 2008)

13. D. Childs, C. Carter, Rotordynamic characteristics of a 5-pad, rocker-pivot, tilting pad bearing in a load-on-pad configuration; comparisons to predictions and load-betweenpad results, Proc. of ASME Turbo Expo, Orlando, FL, v. 59696, pp. 156-167 (2009)

14. L. San Andrés, Squeeze Film Dampers, Encyclopedia of Tribology, pp. 3252-3263 (Springer, NY, 2013)

15. D. Nelson, L. Hollingsworth, Radial bearing with self-aligning liners, equipped with liquid supports, Probl. of Friction and Wear, Series F, pp. 127-134 (1977)

16. G.A. Messmer, L.A. Branagan, Hydrostatically supported tilting pad journal bearing improvements (Patent USA № 6050727, 1998)

17. A.L. Kiryukhin, G.F. Romanovskiy, V.V. Azhimov, P.V. Cherpita, Diagnostic modeling of ship rotary systems with sliding bearings, KSMA Scientific Works, v. 2 (5), pp. 50-58 (2011)

18. A.L. Kiryukhin, G.F. Romanovskiy, Thermohydrodynamic calculation for sliding bearings of ship propulsion complexes under unspecific operating conditions, Probl. of Tribology, v. 3 (53), pp. 62-71 (2009)

19. A.I. Nakorchevskiy, B.I. Baskok, Hydrodynamics and heat and mass transfer in heterogeneous systems and pulsating flows (Naukova dumka, Kiev, 2001) 